\title{
Beware the steroid responsive nature of a pyrexial illness
}

\author{
S C M Richards, S K M Mukherjee, N D Hopkinson
}

\section{Case report}

A 74 year old Latvian woman presented in April 1992 with a six month history of anorexia, weight loss, and intermittent diarrhoea. She had had a subtotal thyroidectomy in 1983 and 40 years previously she had worked in a tuberculosis sanitorium. She was feverish with bilateral basal crackles on lung auscultation, tender hepatomegaly, and a large asymmetrical thyroid goitre. Baseline investigations showed normal renal, liver, and thyroid biochemistry, but a mild normocytic normochromic anaemia (haemoglobin concentration $11.3 \mathrm{~g} / \mathrm{dl}$ ) and an erythrocyte sedimentation rate (ESR) of $80 \mathrm{~mm} / 1 \mathrm{st} \mathrm{h}$. Chest radiography revealed bilateral mid and lower zone shadowing consistent with pulmonary fibrosis. Abdominal ultrasound examination was normal and blood and urine cultures (including early morning urine for acid and alcohol fast bacilli (AAFB)) were negative. Thyroid microsomal antibodies and rheumatoid factor were positive at titres of $1: 400$ and 1:40, respectively. Thyroid ultrasound examination showed a large thyroid gland with retrosternal extension and cervical lymphadenopathy. As the patient remained feverish, a working diagnosis of De Quervain's thyroiditis was made and she began taking steroids. Her pyrexia settled and she was discharged home on a reducing course of prednisolone.

Two months later, the patient fell onto her right hip. Radiology showed no fracture. However, over the next month she had worsening pain in her right leg down to her knee, with difficulty in weight bearing. There were no clinical features of deep vein thrombosis and venography was normal. She was noted to be febrile again and sputum cultures grew Staphylococcus aureus which was treated with oral flucloxacillin. As her fever failed to settle steroids were again introduced.

In August 1992 the patient was readmitted to hospital because of difficulty in weight bearing. She had lost $7 \mathrm{~kg}$ in weight since her initial presentation. Rheumatological review revealed tenderness over the right trochanteric bursa and a right knee effusion with lack of full knee flexion. Bone scintigraphy showed only a mild increase in activity around the right knee joint. As aspiration of the knee was sterile, the joint was injected with depomedrone. Whilst still an inpatient, she developed swelling over the anterolateral aspect of the right thigh and a fever returned. Computed tomography (CT) scan of the thigh showed increased density in the subcutaneous fat consistent with inflammation, but the underlying bone and muscles were normal'. Attempted aspiration of the area was unsuccessful. Further investigation into the cause of the pyrexia showed hypergammaglobulinaemia (IgG 28.1 g/l), normal liver biopsy including culture for AAFB, and a borderline positive Heaf test (grade 2-3). A working diagnosis of an infected haematoma was made and oral clindamycin and ciprofloxacin therapy was started. The patient's fever persisted so her steroids were increased further, with the result that her temperature returned to normal and her leg pain and swelling improved, allowing her to return home.

Over the next few months the patient developed several osteoporotic vertebral fractures and her steroids were reduced and eventually stopped. Cyclical etidronate was commenced. In August 1993 she was anticoagulated for pulmonary embolism (high probability on ventilation/perfusion lung scanning) and, because there was further fever, steroids were again introduced with good effect. The recurrent right thigh swelling with a good response to prednisolone led the clinical team in charge of the patient to suggest that this may be a localised form of myositis, though serum creatine kinase was normal and muscle biopsy was never done.

In May 1994, she presented with worsening swelling of her right thigh (now present for two years), and examination confirmed fluctuance and erythema. The differential diagnosis included a large abscess or muscle tumour. Magnetic resonance imaging (MRI) (figure) showed a massive trochanteric bursa extending deep into the thigh, with rupture through tensor fascia lata. Aspiration yielded pus that grew Mycobacterium tuberculosis. A total of $500 \mathrm{ml}$ was drained surgically and antituberculosis treatment was commenced. The patient made a good recovery.

\section{Discussion}

Mycobacterial infections of bursae, joints, and bones (skeletal tuberculosis) present insidiously with a slow evolution of physical findings, often intially with normal radiological investigations. ${ }^{12}$ The first symptom is typically pain $^{3}$ and the natural history may be slow progressive destruction with spontaneous exacerbation and remission of symptoms. Delay in diagnosis is common, with a mean interval from onset of symptoms to diagnosis 

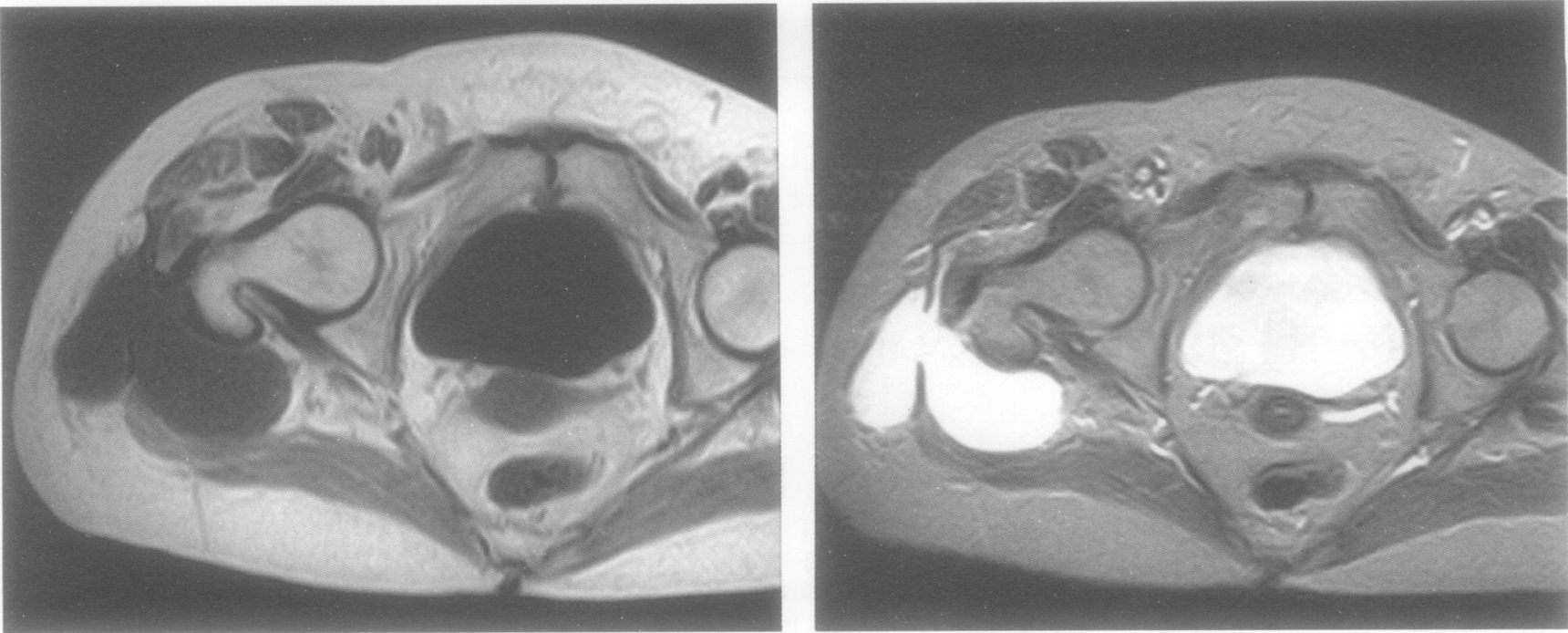

$T 1$ (left) and T2 (right) weighted transverse MRI of the pelvis, demonstrating a fluid filled greater trochanteric bursa. This extends through fascia lata into the subcutaneous fat within the lateral aspect of the thigh, subsequently tracking inferiorly.

of 19-20 months. ${ }^{13}$ Steroids contribute significantly to such a delay in diagnosis by giving temporary relief from symptoms. ${ }^{23}$

The right upper quadrant tenderness with normal abdominal ultrasound examination in this patient might have suggested pleuritis. However, careful review of the radiology suggested that the patient probably never had pulmonary tuberculosis, although the pulmonary fibrosis seen on the chest radiograph did cause diagnostic confusion. This fibrosis, together with an increased ESR, hypergammaglobulinaemia, and positive rheumatoid factor, gave the impression that she had an undifferentiated connective tissue disease, and hastened the erroneous diagnosis of myositis Positive rheumatoid factors are known to occur in both fibrosing alveolitis and tuberculosis. ${ }^{45}$ In De Quervain's thyroiditis, low titre antithyroid antibodies occur (as in this patient), but rheumatoid factors are not specifically associated. ${ }^{6}$ Unfortunately in this patient, the mild anaemia gave no clue to the chronicity of her underlying disease. Importantly, CT scan of the thigh in 1992 was non-specific and, in retrospect, magnetic resonance imaging would have been the investigation of choice.

Skeletal tuberculosis accounts for approximately $1 \%$ of all patients with tuberculosis, $50 \%$ of whom have evidence of coexistent pulmonary disease. ${ }^{7}$ In the recent literature ( $\leqslant 15$ years) tuberculous bursitis appears to be unusual, with 10 cases of olecranon and one of ischiogluteal bursitis. ${ }^{8-11}$ Only one previous case of tuberculous trochanteric bursitis could be found. ${ }^{10}$ To facilitate diagnosis, a positive Heaf test (grade 3-4) is of value, but this test may be negative, especially in those with an impaired cell-mediated response. Suspicion of a tuberculous lesion should be confirmed by microscopy and cultures and, if this is unsuccessful, by appropriate biopsy.

\section{The lesson}

- A high index of clinical suspicion is needed to make the diagnosis of skeletal tuberculosis infection. Delay in diagnosis is common, and as steroids may temporarily produce good symptom relief, the need for bacteriological or histological confirmation, or both, is essential.

We wish to thank Dr G Sommers for providing the MRI prints.

1 Davidson P T, Horowitz I. Skeletal tuberculosis: a review of patient presentations and discussion. $\mathrm{Am} \mathrm{f}$ Med 1970; 48: $77-84$.

2 Parkinson R W, Hodgson S P, Nobley J. Tuberculosis of the elbow: a report of five cases. $\mathcal{F}$ Bone foint Surg Br 1990 72: 523-4.

3 Walker G F. Failure of early recognition of skeletal tuberculosis. BMF 1968; i: 682-3.

4 Turner-Warwick M, Burrows B, Johnson A. Cryptogenic fibrosing alveolitis: clinical features and their influence on survival. Thorax 1980; 35: 171-80.

5 Holborow E J. Autoantibodies in the rheumatic diseases In: Scott J T, ed. Copeman's Textbook of the Rheumatic Diseases, 6th edn. Edinburgh: Churchill Livingstone, $1986 ; 358$.

6 Greene J N. Subacute thyroiditis. Am $\mathcal{f}$ Med 1971; 51: 97-108.

7 US Department of Health, Education and Welfare Centre for Disease Control tuberculosis statistics in the US. for Disease Control tuberculosis statistics

8 Mishriki Y, Langan P. Isolated olecranon bursitis of tuberculous origin. Orthop Rev 1984; 13: 89-92.

9 Maloney J M, Gregg C R, Stephens D S, Manian F A Rimland D. Infections caused by Mycobacterium szulgai in humans. Rev Inf Dis 1987; 6: 1120-6.

10 Butorac R, Littlejohn O, Hooper J. Mycobacterium disease in the musculoskeletal system. Med $\mathcal{F}$ Aust 1987; 147: 388-91.

11 Chafetz N, Genant H K, Hoaglund F T. Ischiogluteal tuberculous bursitis with progressive bony destruction. Can Assoc Radiol f 1982; 33: 119-20. 